\title{
DICTIONNAIRES ET TRADUCTION DE ROMANS CONTEMPORAINS
}

\author{
Jean-Louis Vaxelaire \\ jean-louis.vaxelaire@unamur.be \\ Université de Namur (Belgique)
}

\section{Résumé}

Par leur caractère polyphonique, de nombreux romans contemporains posent des problèmes lexicaux au traducteur en mélangeant lexique standard, argot et termes techniques. La question qui se pose est alors de savoir si les dictionnaires peuvent être utiles au praticien. Nous verrons que pour des raisons théoriques et pratiques, l'aide qu'ils apportent est limitée, un dictionnaire réellement utile devrait changer ses présupposés conceptuels, donc devenir un dictionnaire culturel et adopter une forme électronique.

\begin{abstract}
"Dictionaries and translation of contemporary novels"

Lexical problems do arise in many contemporary novels because of their polyphonic nature, mixing standard words, slang and technical terms. We must then ask ourselves if dictionaries may be useful to assist translators. We will see that for theoretical and practical reasons, this assistance is limited; a really useful dictionary should change its conceptual assumptions, therefore becoming a cultural dictionary in electronic form.
\end{abstract}

Mots-clés : Dictionnaire. Traduction. Sens. Culture.

Keywords: Dictionary. Translation. Meaning. Culture. 



\section{Introduction}

Lattitude des traducteurs face aux dictionnaires est très variée ; entre celui qui s'en sert comme d'un outil de vérification nécessaire et celui qui refuse de l'utiliser sous le prétexte qu'il est parfaitement bilingue, toutes les pratiques semblent exister. Au vu du thème de ce numéro, il sera plus utile de se placer du côté des traducteurs qui ont recours, que ce soit ponctuellement ou couramment, à un dictionnaire. Les questions qui se posent alors relèvent de plusieurs champs : du point de vue de la traductologie, les dictionnaires apportent-ils une amélioration sur le plan de la qualité et/ou offrent-ils un gain de temps au praticien? Du point de vue de la lexicographie, est-ce qu'un type de dictionnaire (monolingue ou bilingue, de langue ou encyclopédique, etc.) serait préférable pour le traducteur? Enfin, du point de vue de la sémantique, les choix théoriques des lexicographes devraient-ils être modifiés pour mieux répondre aux attentes des traducteurs?

Je partirai également du principe que la probabilité de ne rencontrer que des textes aisément interprétables est faible. Leech \& Short écrivaient à propos d'un titre de journal : " imagine the bafflement of a reader who spoke English, but had spent all his life in an alien environment in another galaxy » (2007 : 123). Est-il vraiment nécessaire d'imaginer un scénario aussi complexe : prendre comme exemple un hispanophone ou un francophone qui a une bonne connaissance de la langue anglaise mais qui ne comprend pas le sous-entendu du titre est suffisant. Lorsque l'on voyage, on s'aperçoit que des collègues étrangers parfaitement bilingues ont parfois du mal à comprendre les titres du Canard Enchaîné car ils vivent loin du pays d'origine de ce journal. Certains titres sont même compliqués pour des francophones d'autres pays (le Canada par exemple) tellement les références peuvent être spécifiques à la réalité sociale française. Nombre de romans actuels posent des problèmes lexicaux en mélangeant différents registres, par exemple les policiers qui, pour accentuer leur réalisme, vont intégrer des termes techniques ${ }^{1}$ mais aussi

1. Par exemple, dans cet extrait :

"He Ex-laxed it,' Kincaid announced. 
des éléments plus relâchés avec un certain nombre de mots d'argot. Puisque l'on est amené quotidiennement à employer des registres distincts selon les situations, ces romans exploitent des ressources lexicales si diversifiées qu'il est difficile de toutes les maîtriser, même dans sa langue maternelle.

\section{Le traducteur et le dictionnaire}

\subsection{Le dictionnaire en tant qu'allié du traducteur}

Après qu'un étudiant lui avait demandé lors d'une conférence quel type de dictionnaire il utilisait, un traducteur confirmé avait affirmé qu'il n'en employait jamais, qu'il maîtrisait suffisamment les deux langues pour s'en passer. On peut certes le croire, mais il est toutefois difficile de ne jamais rencontrer un mot que l'on maîtrise peu ou mal dans un texte de sa propre langue maternelle. Dans ma pratique de traduction (de l'anglais vers le français), j'ai à de nombreuses reprises dû me référer à un dictionnaire pour des termes techniques, des mots d'argot ou des sigles que je ne connaissais pas. Plutôt que de rester dans le flou ou de faire une erreur, le recours à un dictionnaire semble la meilleure solution.

Les traducteurs techniques peuvent probablement connaître tous les termes de leur domaine au bout de quelques mois ou de quelques années, mais ils ont dû passer par une phase d'apprentissage pour laquelle le dictionnaire peut s'avérer un bon allié.

Les traducteurs de romans se retrouvent peut-être dans une situation plus complexe aujourd'hui. En effet, il n'y a pas de lien lexical ou stylistique d'un roman à un autre, et parce que le réalisme est une tentation répandue, de nombreuses ouvres contiennent des éléments techniques peu connus : des termes de médecine légale dans tel roman policier, des concepts philosophiques ou des éléments liés au vocabulaire du jardinage dans tel autre livre. La dimension polyphonique du roman n'est pas toujours évidente même pour le locuteur natif de la langue source, l'ouvrage peut jouer sur les registres de langue ou les champs lexicaux les plus divers. Si le livre à traduire contient de nombreux termes de la marine à voile et que le traducteur ne les connaît

\footnotetext{
'How's that?' Sellito called.

'Used disappearing ink. We call it Ex-laxing in the business. The old Ex-lax contained phenolphtalein. Before it was banned by the FTA. You'd dissolve a pill in alcohol and make a blue ink. It had an alkaline pH. Then you'd write something. After a while, exposure to the air would make the blue disappear.'

[...] 'Exactly. You don't see phenolphtalein much anymore. But you can do the same thing with thymolphtalein indicator and sodium hydroxide." (Deaver, The Vanished Man, Philadelphie, Coronet Books, 2003).
} 
que peu ou mal dans sa langue maternelle, l'usage d'un dictionnaire spécialisé devient indispensable.

Lorsque le signifié de la lexie n'est pas lexicalisé dans la langue cible, les dictionnaires bilingues offrent en règle générale une glose souvent plus intéressante qu'une simple équivalence, et les monolingues permettent de mieux saisir ce qui peut être difficile à comprendre. Dans un livre contre l'anglais international, Hagège (2012) regrette que deux termes danois soient traduits par un même nom anglais. Ainsi, førtidspension renvoie à une pension obtenue après décision collective de faire partir avant terme les personnes atteintes d'incapacité alors que efterløn correspond à un départ volontaire soumis à une cotisation du salarié. Dans les deux cas, l'anglais traduit par early retirement ce qui masque les spécificités de la protection sociale danoise. Néanmoins, un bon dictionnaire bilingue anglais-danois indiquera ces spécificités et les monolingues danois permettront au traducteur de bien distinguer ces deux mots.

Parmi les approximations que l'on peut recenser dans des traductions, plusieurs auraient pu être évitées avec l'aide d'un simple dictionnaire bilingue. Ainsi, dans Black Album de Kureishi (Londres, Faber \& Faber, 1995 pour la version anglaise et Bourgois, Paris, 1996 pour la traduction française), le terme disques de contrebande pour bootleg records est un mauvais choix, le Robert \& Collins indique que lorsqu'on parle de document (livre, disque, etc.), il faut employer l'adjectif français pirate pour traduire bootleg. De même, dans un autre ouvrage du même auteur, Intimité (Paris, Bourgois, 2000), on lit : « ils fumaient du hasch, de la "merde" comme ils disaient » (p. 31). D'après le Robert \& Collins, dans le domaine de la drogue, shit demeure shit en français.

\subsection{Parce qu'un dictionnaire ne peut pas tout faire}

La France est un pays où le culte du dictionnaire est répandu dans les milieux scolaires. Si l'école est laïque, il arrive que le dictionnaire devienne une nouvelle forme de bible (le Dictionnaire encyclopédique Quillet de 1953 tient un discours de ce type : «Pendant des millénaires, les sources du savoir furent des livres sacrés ; ce sont aujourd'hui des dictionnaires. Aux livres sacrés, les peuples ont demandé la révélation ; au dictionnaire, ils demandent la connaissance. ») et les professeurs répètent régulièrement aux parents qu'il est nécessaire d'avoir un dictionnaire à son domicile, on pense souvent au Petit Larousse pour les premières années puis au Petit Robert pour le lycée.

Toutefois, si le consensus existe pour la langue maternelle, le dictionnaire peut parfois, d'après certains professeurs de langue étrangère, devenir le pire ennemi de l'élève. J'ai eu personnellement l'occasion de voir il y a près d'une dizaine d'années une copie rédigée par un collégien pour un cours d'anglais à 
l'aide d'un dictionnaire bilingue français-anglais : l'élève avait écrit son texte en français puis avait traduit littéralement chaque mot par un terme anglais (avec à plusieurs reprises des erreurs liées à l'homonymie). Ce travail, qui avait dû lui prendre énormément de temps puisqu'il avait vérifié chaque mot dans le dictionnaire, était strictement illisible pour un anglophone et ne le devenait partiellement pour un francophone qu'en essayant de retraduire en français. Dans ma pratique d'enseignement avec des étudiants étrangers, je les ai constamment mis en garde face aux dictionnaires de synonymes qui leur semblent être des outils pour améliorer leur lexique. En effet, ce type d'ouvrage n'est employable que lorsqu'on maîtrise parfaitement la langue, les contresens sont sinon particulièrement dangereux : le dictionnaire de synonymes de Word me propose ainsi intrépide pour remplacer dangereux. Un francophone sait parfaitement que des contresens ne peuvent être "intrépides ", mais ce n'est pas le cas de tous les étudiants qui apprennent le français.

Bien que l'on s'éloigne des situations d'apprentissage avec la traduction automatique, sa faillite patente me semble relever de la même problématique: les premiers promoteurs de la traduction automatique sont partis du principe qu'avec un dictionnaire d'équivalences et quelques règles de syntaxe, on arriverait à se passer des traducteurs et que tous les textes seraient accessibles dans sa langue maternelle. Leur constat d'échec démontre que le dictionnaire ne peut tout résoudre. La raison principale tient au principe même des ouvrages lexicographiques : le dictionnaire tel qu'il existe ne peut présenter que des lexies décontextualisées ${ }^{2}$ alors que les textes qui sont lus ou traduits apparaissent évidemment dans un cadre particulier, ils relèvent d'un genre et d'un type de discours. Grass (2002 : 74) donne un exemple allemand qui semble difficilement traitable automatiquement :

(1) Paul fährt einen Marder.

D'après Grass, un ordinateur, parce qu'il ne dispose d'aucune connaissance du monde, proposera une traduction mot à mot :

(2) * Paul conduit une martre.

Les humains qui utiliseraient le même dictionnaire bilingue ne choisiraient pas cette solution absurde, mais pour trouver une traduction satisfaisante, ils devraient savoir que l'on se situe dans un discours lié au fait militaire car le Marder est un véhicule blindé de reconnaissance de l'armée allemande. Parmi

2. Meschonnic (2008: 16) juge que les bons dictionnaires sont ceux qui présentent des citations littéraires, des citations qui ont justement pour but de réintroduire un contexte particulier. 
les sites de traduction automatique (testés le 07/05/2013), Voila et Linguatec proposent la phrase (2), Systran également mais avec une syntaxe très étrange (Conduit Paul une martre), Reverso aboutit à un résultat impossible en français en choisissant un verbe différent (Paul va une martre), InterTran ne possède pas tous les termes dans son dictionnaire ce qui donne une phrase sans queue ni tête (Paul charrettes on Marder), enfin Google traduction et Bing modifient le verbe pour que celui puisse se combiner avec l'argument, mais de la correction syntaxique découle une incorrection sémantique : avec le premier, Paul entraîne une martre ${ }^{3}$ et dans le second, Paul exécute une martre ! Le résultat n'est d'ailleurs même pas probant car si on peut entraîner des chiens ou des ours dans un cirque, on n'entraîne jamais à ma connaissance des martres, quant à les exécuter, c'est également étrange puisque l'exécution est réservée aux êtres humains, cette occurrence ne serait donc possible que dans le discours d'un défenseur des animaux.

Léchec découle en priorité d'une approche théorique déficiente : comme l'avait annoncé Saussure il y a plus de cent ans, une langue n'est pas une nomenclature, mais ce n'est pas non plus une nomenclature agrémentée de quelques règles combinatoires. Lidée qu'un dictionnaire pourrait servir à réaliser une traduction ne tient pas compte d'une opposition essentielle en linguistique qui est celle entre la signification et le sens. Parce qu'il dépend d'une théorie du signe, le dictionnaire tente de définir la signification des lexies, alors que le traducteur, dont le travail relève d'une théorie du texte, cherche à retranscrire le sens des énoncés originaux ${ }^{4}$ (sur la distinction entre théorie du signe et théorie du texte, voir Rastier 1994). Pour le dire autrement, la lexicographie étudie in vitro les termes alors que la traduction le fait in vivo. Signification et sens sont évidemment très liés, mais l'influence des contextes particuliers sur le second fait que le dictionnaire n'est pas toujours pertinent. C'est en raison du principe que le global détermine le local que l'on demande

3. Puisque les choix de conservation ou de modifications d'éléments dans Google traduction dépendent d'algorithmes, ils sont imprévisibles. Ainsi, selon les contextes, Guillaume peu devenir William. Par contre, j'ai découvert un cas où la traduction est globalement réussie : dans l'énoncé « les femmes devenaient Mme Henri Dupont ou Mme Jean Martin en se mariant ", les noms propres sont employés comme exemples de noms banals en français, ce que l'on retrouve dans la traduction proposée : « the women became Mrs. Henry Smith or Mrs. John Smith by marriage ».

4. Béjoint résume avec des termes différents le problème qui se pose pour le traducteur : « le dictionnaire traditionnel est mal armé pour répondre aux questions des utilisateurs qui portent sur la compréhension des actes de discours, dans la mesure où il traite d'unités de la langue alors que l'utilisateur aurait besoin de voir traiter des fragments de discours. » (Béjoint 2005 : 16). 
aux étudiants de lire entièrement un texte avant de commencer à le traduire : un même terme pourra prendre des sens différents selon le contexte général et la connaissance du projet artistique de l'auteur influencera le choix de traduction des passages.

\section{Qualité et gain de temps}

En ce qui concerne la traduction humaine, le point principal est d'ordre pratique. On sait qu'aujourd'hui, on laisse de moins en moins de temps aux traducteurs pour accomplir leur tâche, il faut donc se demander si un dictionnaire peut apporter une aide réelle, offrir par exemple un gain de temps pour terminer le travail qui doit être accompli.

\subsection{Le cas des noms propres}

J'ai démontré dans d'autres travaux (Vaxelaire 2005, à paraître) les défauts des dictionnaires dès qu'il s'agit de traiter les noms propres ou leurs dérivés : les dictionnaires de langue tendent à les ignorer ou à les rejeter, et les dictionnaires de noms propres, par leur manque de systématisme et l'insuffisance d'informations linguistiques qu'ils offrent (peu de transcriptions phonétiques, d'indications morphosyntaxiques, etc.), peuvent devenir une source d'erreurs. Ainsi, dans la partie noms propres du Petit Larousse ou dans le dictionnaire des noms propres du Petit Robert, on indique que le nom Beatles est précédé de l'article les en français mais rien n'est écrit à propos du nom Rolling Stones. La conclusion du lecteur est que le second s'emploie sans article en français, ce qui n'est pas le cas : « Les Rolling Stones ont donné un concert surprise samedi à l'Echoplex» (Libération 28/04/13). Ecrire " * Rolling Stones publient un nouveau disque » serait fautif en français car l'article est obligatoire. Le traducteur qui ferait confiance à ces dictionnaires serait par conséquent entraîné à valider plusieurs erreurs. Dans la version française du Bouddha de banlieue de Hanif Kureishi (Paris, Bourgois, 1991), on note un problème de ce type : «mon équipe pourtant était Spurs» (p. 68). Quand bien même le nom du club Tottenham Hotspurs serait dans un dictionnaire - ce qui n'est pas le cas - , il faudrait tracer un lien vers cette abréviation et il n'y aurait probablement pas d'informations sur l'emploi des déterminants. Larticle les est obligatoire dans cet énoncé, une recherche sur Google avec la séquence "les spurs " + foot donne en mai 2013 environ 356000 résultats, ce qui, malgré l'homonymie avec le club de basket de San Antonio, démontre tout de même que les journalistes sportifs ne commettraient pas la même erreur. On ne demande 
évidemment pas à un traducteur littéraire d'être aussi compétent qu'un journaliste sportif dans ce domaine.

Il suffit de lire quelques traductions pour s'apercevoir que le genre des noms propres étrangers n'est pas simple à deviner. Ainsi, La traductrice de Linstinct du langage de Pinker (Paris, Odile Jacob, 1999) écrit ainsi « du NBA » (p. 116) et « la Mayflower» (p. 246) alors que le premier est féminin en français et le second masculin. Les dictionnaires de noms propres oublient pourtant souvent d'indiquer le genre lorsque celui-ci n'est pas référentiel mais lexical. ${ }^{5}$

J'ai noté dans plusieurs travaux que la traduction des noms propres pouvait être perçue comme un problème complexe lorsqu'on l'observait dans sa diversité : les critères qui amènent le praticien à conserver ou à modifier les noms propres dans son texte sont nombreux (Vaxelaire 2006, 2011). Les dictionnaires bilingues peuvent fournir des informations à ce sujet puisque les principaux noms de lieux par exemple apparaissent dans les nomenclatures. Les deux noms non modifiés de la page 107 de la version française du Transport de A.H. de George Steiner (Paris, Julliard/LÂge d'Homme, 1981) sont toutefois deux cas différents. Si le premier aurait dû être changé selon le Robert \& Collins ( le consul général d'Ecuador» du livre doit être remplacé par « le consul général d'Equateur »), le second n'est pas recensé dans ce dictionnaire : le traducteur écrit que " l'express de nuit venant d'Oporto, le 9 h14, ralentissait, avant d'entrer à Lisbonne ", mais le Robert \& Collins ne connaît pas cette forme anglaise plus ancienne du nom de la ville de Porto et ne peut aider.

Les noms les plus récents risquent eux aussi d'être absents des dictionnaires bilingues. Ainsi, les noms de partis politiques italiens sont généralement traduits en français (les dernières élections législatives de 2013 ont vu entre autres s'opposer le Parti démocrate, le Peuple de la Liberté, le Mouvement 5 étoiles, Agir pour arrêter le déclin ou Choix civique avec Monti pour l'Italie), mais si on lit un article du Monde du 11/04 /06, on notera que tous les noms de partis sont traduits (Ligue du Nord, Flamme Tricolore, L'Olivier, Italie des Valeurs, etc.) à l'exception de Forza Italia. Je n'ai trouvé aucun journal donnant uniquement le nom français, il apparaît parfois entre parenthèses avec une glose indiquant que ce nom se traduit littéralement par « Allez l'Italie ». Puisqu'il n'y a aucune prédictibilité derrière ce choix de conserver la forme originale (Partito Democratico est plus transparent pour un francophone que

5. Dahl (2000) distingue genre référentiel (déterminé par le référent qui sera du genre masculin ou féminin) et genre lexical (déterminé sur la base des propriétés du nom). 
Forza Italia, il a pourtant été traduit), le dictionnaire bilingue devrait fournir ce type de noms, mais quelquefois ceux-ci naissent et disparaissent d'une élection à l'autre, ce qui n'est pas gérable avec des dictionnaires papier.

\subsection{Le lexique des marges}

Le dictionnaire peut être extrêmement utile en tant que prêt-à-parler pour reprendre l'expression de Collinot et Mazière (1997), c'est-à-dire en présentant des marques d'usage qui aident le traducteur à bien saisir le registre choisi par l'auteur. Malheureusement, un travail que j'avais réalisé lorsque j'étais étudiant (Vaxelaire 1998) établissait que le traitement des mots d'argot divergeait d'un dictionnaire à l'autre : la nomenclature n'était pas identique et les degrés de registre pouvaient être distincts. Par exemple, politico se voit associé à la marque derogatory (qui est généralement associée aux termes racistes ou sexistes) dans le New Shorter Oxford English Dictionary alors qu'aucune n'est indiquée dans le Longman Dictionary of Contemporary English. Quelle attitude doit donc adopter le locuteur non-anglophone vis-à-vis de ce terme? Peut-il l'employer sans crainte (Longman) ou doit-il être très largement évité (Oxford)?

Les termes liés au sexe, pourtant extrêmement nombreux en argot, tendent à ne pas apparaître dans les dictionnaires qui sont avant tout des objets commerciaux; on sait pourtant que la littérature contemporaine n'hésite pas à employer ce genre de mots.

La différence de traitement entre les ouvrages me paraît liée à un problème classique de la lexicographie : le manque de mise à jour. L'évolution des degrés de vulgarité des mots d'argot est parfois très rapide, en une génération un terme grossier peut devenir familier, mais les dictionnaires peinent à suivre cette évolution.

Lorsque le narrateur de Black Album parle de " a couple of Goths » (p. 112), la traductrice suit le dictionnaire bilingue (Larousse par exemple) en écrivant : « deux Goths » (p. 157). Puisque l'action se situe dans la Londres contemporaine, où des noms de groupes punks ont été cités quelques paragraphes auparavant, il aurait mieux valu traduire par gothiques sans majuscule puisque l'on parle du mouvement musical et non du peuple germanique. Les éléments correspondants aux sous-cultures apparaissent peu dans les dictionnaires.

Dès que l'on aborde l'argot ou les sous-cultures, on se situe aux marges du lexique standard. Les régionalismes se situent également dans cette zone que les dictionnaires ont du mal à aborder ou se refusent à traiter. Le roman Texaco de Patrick Chamoiseau a obtenu le prix Goncourt 1992, mais cet écrivain, 
parce qu'il n'hésite pas à proclamer sa créolité, a opté pour l'emploi de termes typiques des Antilles. Rien que dans les trois premières pages, on note «l'Enville » (p. 19), " achélèmes », " ces huissiers des vieux temps-la-misère », « sa manman », « sous la pluie des boutous » (p. 20), « Iréné comprit flap », " quelque chien-fer galeux vestimenté en homme », "Iréné rejoignit son gommier », « un jeune braille à locks » (p. 21). Certaines lexies sont transparentes en contexte mais pas toutes pour un francophone non habitué au créole antillais. L'emploi d'un dictionnaire de langue français sera inutile car la place allouée aux régionalismes est très faible, les dictionnaires de langue sont avant tout ceux du français métropolitain normé. Au-delà de ces formes qui n'apparaissent pas dans les nomenclatures, on peut également se demander si le mot "marâtre » (p. 20) est employé comme en français standard (il est indiqué « vieilli » d'après le Petit Robert) ou dans un sens régional qui serait différent. Sur ces questions, les bilingues sont encore moins utiles car la part des régionalismes semble encore plus faible : les étrangers sont obligés de plus s'intéresser au français de Paris qu'à celui de la Belgique ou du sud de la France.

\subsection{Une divergence d'unités}

Il est évident que de nombreux choix de traduction ne concernent pas du tout le dictionnaire : si l'on souhaite conserver dans le texte cible un jeu de mots, les modifications porteront sur des unités plus larges que la lexie. Lunité du dictionnaire est généralement le mot graphique alors que le traducteur fonctionne avec des passages plus ou moins longs. Ces passages dépendent du texte intégral, mais aussi d'un corpus plus important : les autres œuvres du même auteur, le genre dans lequel s'insère l'ouvrage, ${ }^{6}$ les relations que l'œuvre peut avoir avec d'autres, etc.

La dimension historique ne doit pas non plus être oubliée. Si l'on doit retraduire des romans, ce n'est pas nécessairement parce que les premières traductions étaient de mauvaise qualité, mais parce que chaque traduction

6. Cette remarque est aussi valable pour les noms composés : bien que Seelöwe se traduise par otarie en français, presque personne n'emploie Opération Otarie pour Unternehmen Seelöwe, le plan d'invasion allemand du Royaume-Uni pendant la Seconde Guerre mondiale. On lui préfère un mélange français-allemand Opération Seelöwe ou une traduction littérale Opération Lion de Mer. Bien que les traductions mot à mot soient vouées aux gémonies, celle-ci est bien meilleure dans le cadre des noms d'opérations militaires : l'otarie est pour un francophone un animal sympathique que l'on voit dans les cirques, le nom du « lion de mer » a par contre une dimension plus menaçante, qui convient donc nettement mieux au rôle qui lui est attribué. 
s'inscrit dans une époque et un système de valeurs. Ce qui correspond aux normes du XIXe siècle ne correspond plus à celles du siècle suivant. Pour ne prendre qu'un exemple, Podeur (1999: 76) donne celui de la première traduction française d'Il Piacere de Gabriele d'Annunzio en 1889 (par G. Hérelle) et d'une seconde version qui n'est en fait qu'une correction de cette première traduction (par P. de Montera en 1971).

L'extrait original est le suivant :

Andrea Sperelli aspetteva nelle sue stanze un'amante. [...]

Non era Elena; ma una signora che voltò per la via Gregorianna, camminando adagio.

La traduction de 1889 :

André attendait Helène Muti. [...]

Mais ce n'était pas Hélène ; c'était une dame qui, d'un pas lent, tourna par la rue Grégorienne.

La version corrigée de 1971 :

Andrea Sperelli attendait chez lui sa maîtresse. [...]

Mais ce n'était pas Elena ; c'était une dame qui, d'un pas lent, tourna par la via Gregorianna.

Dans cet extrait, la correction joue principalement sur un rejet de la naturalisation effectuée au XIX $X^{e}$ siècle. À notre époque, il n'est plus utile, on pourrait même dire défendu, de franciser les noms italiens, alors que cela semblait tout à fait naturel au XIX siècle.

Les nouvelles traductions, parce qu'elles ne sont pas une simple correction d'une première version, offrent souvent des modifications plus radicales (on pensera à celles de Dostoïevski par André Markovicz qui a essayé de rendre un style plus relâché en français). ${ }^{7}$ On pourra toujours répondre que les dictionnaires évoluent eux aussi, ce qui est vrai, mais nous connaissons tous des personnes qui continuent à travailler avec les premières éditions du Littré, celui-ci ayant la réputation de proposer un lexique plus riche.

\section{Quelle lexicographie pour le traducteur ?}

\subsection{Dictionnaire et culture}

Outre la divergence entre la théorie du signe qui régit le dictionnaire et la théorie du texte dont dépend la traduction, l'autre problème principal des

7. Markovicz a reproché aux premiers traducteurs d'avoir « amélioré » Dostoïevski pour le rapprocher des normes françaises du XIX ${ }^{\mathrm{e}}$ siècle, ce qui a eu pour effet de dénaturer ses œuvres. 
ouvrages lexicographiques est leur manque de profondeur culturelle. Cette dimension est de plus en plus prise en compte (les éditions récentes du bilingue Robert $\mathcal{E}$ Collins ont ainsi intégré des encadrés culturels à propos de lexies ou d'expressions), mais il existe encore un énorme déficit à ce sujet.

Un exemple tiré de Black Album de Kureishi (pp. 111-112) l'illustre bien lorsqu'un ancien toxicomane fait un récapitulatif de ses journées : il commençait avec des restes de cocaïne, fumait un joint, buvait une bouteille de cidre avant de prendre de l'ecstasy, de l'acide, pour terminer avec une intraveineuse. Dans la traduction française, le terme cidre introduit une rupture d'isotopie car il n'est pas logique d'associer le cidre, boisson très peu alcoolisée que l'on boit avec des crêpes (que l'on voit même parfois proposée à des enfants), et des drogues dures pour la plupart. Les dictionnaires bilingues proposent pourtant pour équivalent du cider anglais le mot français cidre. Les ouvrages monolingues ne sont pas plus utiles, si l'on excepte la Cambridge Encyclopedia qui indique que le degré d'alcool va de 3 à $9 \%$, ce qui indique une différence avec le cidre français. C'est en effet ce degré d'alcool qui marque une distinction nette entre les deux boissons qui, si elles portent le même nom, ne sont pas identiques et leur nom n'apparaît donc pas dans les mêmes contextes : on peut associer cider et alcoholism dans un texte anglais, ce qui semble difficilement possible en français avec cidre et alcoolisme. Les dictionnaires se limitent à une description du référent au lieu de donner des informations plus culturelles et ne permettent donc pas de bien saisir le sens de ce passage. ${ }^{8}$

Ce constat a déjà été établi par Robert Galisson dans le cadre de la didactique des langues. Ainsi, à la lecture de l'article dragée dans un dictionnaire de langue monolingue français, on apprend quels sont les ingrédients qui la composent, « en revanche, on ne nous dit pas que les dragées accompagnent toujours la cérémonie du baptême » (Galisson 1991 : 122). La composition d'un objet relève de l'encyclopédique qu'est censé rejeter le dictionnaire de langue, alors que la dimension culturelle (le rôle symbolique des dragées dans la culture française) est ignorée. À l'exception des documents liés à la fabrication de la confiserie, les textes à traduire contenant le mot dragée feront évidemment plus référence à la place culturelle des dragées qu'aux ingrédients qui les composent. La seule manière de réintégrer du culturel est de passer,

8. Le cas est différent car il concerne le lecteur et non le traducteur : quand un personnage du même roman compare le frère du héros à Saturday Night Fever, il faut savoir que ce film est l'apogée de la période disco et de ses paillettes. Les dictionnaires de cinéma donnent des informations techniques, un résumé mais pas le contexte culturel qui permettrait au lecteur d'interpréter cette image. À nouveau, l'approche est toujours bien plus référentielle que culturelle. 
à l'instar du TLF, par les citations littéraires, comme celle de Camus (« À la naissance de sa fille, il y avait eu des dragées pour tout le monde »).

Galisson regrette que les dictionnaires opposent la culture savante (représentée par les abondantes citations d'auteurs légitimés) et la culture partagée qui est oubliée. C'est pourtant souvent de cette dernière qu'ont besoin ceux qui étudient une langue étrangère. Galisson a choisi de ne pas réutiliser la «notion fourre-tout de connotation » (Galisson 1991: 128), et lui préfère celle de charge culturelle partagée : " la charge culturelle peut différer selon l'âge, l'origine géographique et l'appartenance socioculturelle des locuteurs. Autrement dit, [... ] dans la C.C.P., il y a de la culture et de la subculture. » (Galisson 1991 : 140). En fait, R. Galisson (1991 : 116) considère que la culture partagée est une culture transversale, contrairement à la culture savante généralement inculquée qui n'est qu'une subculture.

C'est cette charge que l'on retrouve dans des textes et qui est si difficile à saisir quand on ne vit pas dans le pays d'où est issu le texte source (ce qui implique que pour un Français, certains passages de textes québécois peuvent laisser perplexe, bien qu'écrits en français). La distinction entre encyclopédique et culturel implique aussi qu'un même référent peut avoir des charges culturelles distinctes, ainsi, comme l'indique Jean-Claude André, " la carte American Express est la carte de Monsieur Tout-le-Monde aux Etats-Unis, alors qu'elle bénéficie d'une image élitiste en France» (1998 : 278). Ce qui est important pour le lecteur/traducteur étranger d'un texte français, c'est de comprendre que si l'auteur a précisé qu'un de ses personnages a eu une carte American Express, il indique en partie sa position sociale. Un point identique est soulevé par Fabrice Antoine (1999 : 13) à propos de Perrier et Évian aux États-Unis.

Dans le roman Black Album, un personnage d'origine pakistanaise adopté prend des cours d'ourdou, « but when he tried asking for the salt in Southall everyone fell about at his accent » (p. 107). Dans la traduction française, on se limite à l'information générale " demander du sel à Southall », ce qui n'a pas particulièrement d'écho pour un francophone. La consultation de la page Wikipedia de Southall permet d'apprendre que les naissances dans cette ville sont dues à $97 \%$ à des personnes originaires du sous-continent indien, mais il n'existe pas de version française de cette page, et les dictionnaires de noms propres se limitent au factuel. Le lien entre Southall et la forte proportion d'Indiens et de Pakistanais est connue des Britanniques mais pas de tous les lecteurs étrangers. Les rapports entre le Royaume-Uni et le sous-continent indien posent d'autres problèmes de traduction : ainsi, la traductrice modifie « Asian punk band» (p. 113) en " groupe punk pakistanais» (p. 157) alors 
que quelques pages plus loin le chanteur est décrit comme étant « un Indien » (p. 161), ce qui n'est pas forcément compatible avec pakistanais. À l'inverse, le nom «Asia » (p. 178) est rendu par « Asie » (p. 242) alors que Kureishi parle spécifiquement dans ce contexte du sous-continent indien, l'Asie pour les francophones renvoie plutôt à la Chine ou au Japon qu'à l'Inde ou au Pakistan, ce dernier pays étant plutôt associé ces dernières années au monde arabe.

Keith Richards relate dans son autobiographie intitulée Life (Paris, R. Laffont, 2010) une croisière transatlantique et raconte que lui et ses amis s'attendent à voir Noel Coward sur le bateau. Il s'agit évidemment d'une image puisque Coward était mort au moment de cette traversée, mais puisque Noel Coward n'est pas très connu en France, le traducteur opte pour la meilleure solution qui est de proposer une note de bas de page. Cependant, cette dernière semble être un extrait d'encyclopédie et savoir qui était Noel Coward ne sert à rien pour comprendre le contexte : ce qui est important ici ce n'est pas la personne (née à tel endroit, décédée à tel autre, qui a eu telle activité professionnelle), mais sa place dans la culture britannique, car ce que veut faire ressortir Richards, c'est l'ambiance surannée de cette croisière.

Pour prendre un dernier exemple, dans la série norvégienne Hellfjord (diffusée en 2012), Tante Kose, un personnage de grand-mère délurée, dit au héros que les meilleurs films passent après minuit sur Canal Plus. Lallusion au cinéma pornographique est évidente en France où Canal Plus existe, moins dans les pays anglophones où les traducteurs des sous-titres ont choisi : « Best movies on cable start after midnight ». Lhumour de la situation ne réside pas dans la référence à une chaîne en particulier, mais dans le trait culturel partagé qui fait que sur certaines chaînes et à une heure tardive, on diffuse des films pour adultes, ce que rend plus ou moins bien la traduction anglaise dans ce contexte.

\subsection{Des nouveaux supports lexicographiques}

$\mathrm{Du}$ point de vue lexicographique, quel type de dictionnaire pourrait donc être le plus souhaitable pour aider les traducteurs ? On sait que certains traducteurs sont hostiles au dictionnaire bilingue, Mary Snell-Hornby (1988) par exemple, pour qui l'équivalent n'est pas nécessairement ce dont a le plus besoin le traducteur, ce qui paraît évident si le dictionnaire bilingue ne prend pas suffisamment en compte les distinctions de genres, de registres, etc. Il peut cependant être utile pour des points ponctuels tels que l'exemple des noms propres que nous avons vu. Selon leur qualité, les bilingues peuvent rendre des services très différents : ceux qui se limitent à des équivalences mot à mot ne sont pas très utiles, d'autres qui incluent des énoncés figés (proverbes, 
slogans, titres, etc.) le sont beaucoup plus. Il y a eu des efforts pour traiter les phraséologismes mais ces efforts doivent être encore poussés pour rattraper le retard. Un bon dictionnaire doit souvent dépasser la limite du mot graphique car les passages que doit traduire le praticien sont bien plus larges.

Si l'on suit Snell-Hornby, un dictionnaire monolingue ou, selon un modèle qui n'existe pas dans la plupart des pays européens, un dictionnaire monolingue qui présenterait en plus quelques équivalences dans des langues étrangères, est un outil globalement plus utile. La question suivante concerne le support : est-il préférable de choisir un dictionnaire papier ou électronique?

Cette question peut sembler secondaire, elle ne devrait pas l'être car le changement de support offre de nouvelles possibilités aux lexicographes. Malheureusement, de nombreux dictionnaires électroniques ne sont que des numérisations des éditions papier et ne profitent pas des possibilités technologiques. Les seuls services que présentent ces dictionnaires sont alors de pouvoir faire une recherche plein-texte - qui peut parfois être utile - ou d'écouter les retranscriptions phonétiques (ce qui n'est d'ailleurs même pas le cas de tous les dictionnaires électroniques).

Le TLFI est souvent donné comme exemple de passage réussi du papier à l'électronique mais le résultat pourrait sans doute être meilleur. Par exemple, il est possible d'écrire un mot phonétiquement et le dictionnaire se charge d'amener vers la bonne entrée, avec la bonne orthographe : si l'on tape ritme, l'interface propose les entrées rythme, rythmer et rythmé. Si la requête est vais, la première forme conjuguée d'aller au présent, il n'y a pas vraiment de réponse directe, on apprend uniquement que cette forme existe dans une expression qui renvoie à étendre car il y a dans l'entrée de ce verbe une citation « Je vais m'étendre». Plus bas, le site propose toutefois : "Vous trouverez peut-être des meilleurs résultats en essayant un des mots suivants : vais, $\mathbf{v}$, vé, vêt, aller, vêtir. » La solution n'est pas la plus rapide car elle mélange homophonie (le principe qui permet de passer de ritme à rythme) et flexion du verbe (le principe qui permet de passer de vais à aller ou de vêt à vêtir).

Un dictionnaire électronique doit mieux employer les possibilités techniques offertes par ce médium, mais cela passe principalement par une révolution lexicographique qui n'a pas encore eu lieu dans le monde francophone et, d'après ce que j'ai observé, dans les autres cultures. Cette révolution n'a pas eu lieu pour des raisons théoriques (le modèle traditionnel du dictionnaire est ancré dans une théorie du signe multimillénaire et dominante) et pratiques (la gestion des liens hypertextes par exemple nécessiterait des ressources humaines importantes pour vérifier que ces liens sont toujours actifs). Toutefois, lorsqu'on parle de liens, il faut aussi développer les liens internes. Le Petit 
Robert est publié en deux volumes dans son édition papier (langue et noms propres) mais seul le premier a paru en CD-rom. Les deux volumes sont de toute manière conçus séparément et il n'y a pas de liens internes entre les deux. Pourtant, comme le démontre la traduction française de l'Introduction à la sémantique de Tullio De Mauro, il serait utile dans un dictionnaire électronique de permettre des liens entre les adjectifs et les noms dont ils sont dérivés, car il n'est pas évident pour un lecteur français de savoir, quand l'auteur parle de "rhétorique manzonienne et déamicisienne ", qu'il fait référence à De Amicis, et il n'est pas certain que le lecteur sache qui est ce De Amicis.

Dans un roman qui emploie un lexique peu soutenu, il est courant d'utiliser des mots abréviés. Pour une langue comme le français qui utilise principalement l'apocope, la présence d'une liste alphabétique peut être utilisée s'il contient cette abréviation (à l'inverse dans d'autres langues où les aphérèses sont courantes, les listes sont strictement inutiles). Un dictionnaire tel que le TLFI ne propose pas cette possibilité, qui existe par contre dans les versions CD-Rom (et donc payantes) d'autres dictionnaires pour lesquels on peut soit passer par un menu déroulant alphabétique, soit par une requête. Pour autant, même lorsque ces deux manières de consulter sont disponibles, la majorité des variations lexicales recensées dans les corpus sont absents : les abréviations, les orthographes alternatives sont très peu prises en compte puisque le dictionnaire instaure une norme et que ces éléments s'en éloignent. J'avais comparé différents dictionnaires (papier et électroniques, bilingues anglais-français et monolingues anglais) pour un mémoire (Vaxelaire 1998), et un nombre très limité de lexies de mon corpus se trouvaient suffisamment définies pour aider le traducteur. Le caractère figé des dictionnaires ${ }^{9}$ s'op- $^{\prime}$ pose à la créativité des locuteurs d'une langue. Des écrivains contemporains qui cherchent à s'immiscer dans une certaine réalité actuelle (le monde de la musique rock, des drogues, etc.) vont employer des termes qui ne pourront entrer dans les dictionnaires que par leur entremise, car c'est généralement l'écrivain reconnu qui adoube la lexie : c'est parce que tel terme a été employé par un auteur que les lexicographes se sentent permis de l'intégrer.

Contrairement à un dictionnaire papier, un dictionnaire électronique peut être révisé quotidiennement, il est possible d'incorporer un nouveau mot rapidement, ce que réalise un site tel que Wikipédia, mais cela nécessite à nouveau des moyens humains importants. Les contraintes de taille de la

9. Il n'est souvent, pour des raisons économiques, pas possible d'élargir la nomenclature ou de la renouveler. 
nomenclature disparaissent également : les termes d'argot ou de divers domaines techniques n'ont plus de raison d'être rejetés. ${ }^{10}$

Lautre point essentiel est celui de l'unité décrite. Dans un dictionnaire traditionnel, il s'agit du mot, mais ce n'est pas celle du traducteur. Lors de son expérience de lexicographe, Antoine (1999: 17) avait proposé d'intégrer le participe passé humoristique bouillu du dicton « café bouillu, café foutu ", mais son collègue l'avait refusé. Ce rejet découle probablement de la question de l'entrée lexicographique : en se limitant à une entrée lexicale, le dictionnaire classique est obligé de découper les unités polylexicales que sont les expressions, proverbes, etc. Le collègue d'Antoine n'a pas voulu de bouillu car ce lexème n'existe pas en dehors du dicton, si on lui avait proposé de faire entrer directement l'ensemble, sa réponse aurait peut-être été différente. Par le biais du format électronique, il serait simple d'intégrer l'expression et de permettre d'y accéder par un seul des termes dans le champ " requête ».

Le plus grand reproche que l'on peut effectuer aux lexicographes aura été de ne pas avoir assez remis en cause leur pratique par rapport aux possibilités technologiques. Comme l'indique Dziemianko (2012 : 196), un dictionnaire électronique ne devrait pas reprendre un répertoire existant mais être réalisé du début à la fin. ${ }^{11}$ Le constat n'est quand même pas très avantageux pour les dictionnaires car des groupes d'échange et de travail entre traducteurs ont été créés sur Internet et leurs résultats sont d'une grande utilité. Un outil qui recenserait et prendrait en compte ces discussions et propositions serait incroyablement rentable. Des ressources existent, mais il manque soit la volonté, soit les moyens financiers pour réaliser un dictionnaire qui répondrait à des besoins pourtant réels.

\section{Conclusion}

Parce qu'ils relèvent d'une culture, elle-même divisible en dizaines de souscultures, les romans contemporains posent de nombreux problèmes aux traducteurs dès que sont abordés des domaines qu'ils connaissent mal (cela peut

10. La première fois où j'ai entendu le mot d'argot zguègue, qui entre dans la version 2014 du Petit Robert, doit remonter à près de vingt-cinq ou trente ans. La période de probation peut probablement être encore plus longue.

11. Dans le même article, Dziemianko cite quelques études qui ont analysé l'impact du passage au numérique sur les apprenants. D'après l'auteur (2012 : 197), dans un exercice sur les prépositions, on note de meilleurs résultats avec les étudiants qui utilisent la version électronique du Cobuild que chez ceux qui emploient la version papier du même dictionnaire. Toutefois, d'autres études ont des résultats inverses, il est donc difficile de tirer des conclusions sans des travaux à plus large échelle. 
être le rock, le football, la tégestophilie ou la physique nucléaire). Les dictionnaires ont du mal à répondre à ces besoins pour diverses raisons. Les langues principales possèdent certes des dictionnaires monolingues de qualité, mais il n'est pas certain qu'ils soient toujours très utiles pour celui qui doit traduire un texte. Ces dictionnaires permettent de bien apprendre les normes principales d'une langue, mais en règle générale le traducteur les connaît déjà. Dès que l'on aborde des sphères qui s'éloignent de ces normes, et qui ne font que rarement partie de l'enseignement scolaire ou universitaire du traducteur, le dictionnaire classique devient moins intéressant. Que ce soit dans un domaine technique très précis ou avec de l'argot, le traducteur trouvera plus d'informations pertinentes sur Internet, en ciblant bien ses recherches, que grâce aux dictionnaires (il existe toutefois des dictionnaires performants pour certains domaines à l'instar du Grand Dictionnaire Terminologique ou celui d'IATE). C'est là un constat d'échec pour la lexicographie contemporaine.

Une refonte de la pratique lexicographique semble donc urgente car, si l'on observe les dictionnaires français, ils sont tiraillés entre deux pôles qui ne sont pas satisfaisants : d'un côté, une tradition linguistique qui considère la langue comme un système immanent (le dictionnaire de langue pur), de l'autre une tradition référentialiste qui fait preuve d'un réalisme naif ('encyclopédie qui ne s'intéresse qu'à l'objet). Comme le montrait l'exemple de dragée donné par Galisson, ce qui est important pour le traducteur, ce n'est certainement pas que ce soit une amande recouverte de sucre durci, mais sa symbolique dans une culture donnée. Plutôt que d'être de langue ou encyclopédique, le dictionnaire qui serait un bon outil pour le traducteur devrait être culturel.

Enfin, le dictionnaire papier a beaucoup d'avantages (on peut le feuilleter comme n'importe quel livre, le consulter sans se soucier de problèmes informatiques) mais dans une démarche pragmatique (en l'occurrence aider le traducteur), la création d'un outil électronique semble inévitable pour des raisons pratiques : pas de contraintes de taille pour la nomenclature, intégration plus rapide des néologismes, modifications plus simples, possibilité de travailler sur des unités plus larges que la simple lexie, etc.

Des données existent sur Internet (malgré leurs défauts, des sites comme Linguee ou WeBiText peuvent être intéressants car ils présentent des passages entiers) mais elles sont noyées dans la masse. En les regroupant sous un projet précis, il serait sans doute possible de proposer un outil qui réponde aux besoins spécifiques des traducteurs. 


\section{Références bibliographiques}

ANTOINE, Fabrice. (1999) "Lexiculturel, traduction et dictionnaires bilingues." Ateliers 19, pp. 11-18.

BÉJOINT, Henri. (2005) “ Dictionnaires anciens, dictionnaires nouveaux, représentation de la langue et du discours. "Revue française de linguistique appliquée 10(2), pp. 11-18.

Collinot, André \& Françoise Mazière. (1997) Un prêt à parler : le dictionnaire. Paris : PUF.

DAHL, Östen. (2000) "Animacy and the Notion of Semantic Gender. " À: Unterbeck, B. (éd.) 2000. Gender in Grammar and Cognition. Berlin \& New York: Walter de Gruyter, pp. 99-115.

Dziemianko, Anna. (2012) "Why One and Two Do Not Make Three: Dictionary Form Revisited. " Lexikos 22, pp. 195-216.

GALISSON, Robert \& Jean-Claude André. (1998) Dictionnaire de noms de marques courants - Essai de lexiculture ordinaire. Paris : Didier Érudition.

GALisson, Robert. (1991) De la langue à la culture par les mots, Paris : CLE international.

Grass, Thierry. (2002) Quoi ! Vous voulez traduire " Goethe »? - Essai sur la traduction des noms propres allemand-français. Berne : Peter Lang.

HAGÈGE, Claude. (2012) Contre la pensée unique. Paris : Odile Jacob.

LEECH, Geoffrey \& Mick Short. (2007) Style in Fiction: A Linguistic Introduction to English Fictional Prose. Harlow : Longman.

Meschonnic, Henri. (2008) "Le dictionnaire mon trésor. " Préface de G. Dotoli. La construction du sens dans le dictionnaire. Fasano-Paris: Schena-Hermann.

PODEUR, Josiane. (1999) Nomination in azione - il nome proprio nelle traduzioni dall'italiano al francese e dal francese all'italiano. Naples : Liguori.

RASTIER, François. (1994) Sémantique pour l'analyse. Paris : Masson.

Snell-Hornby, Mary. (1988) Translation Studies, An Integrated Approach. Amsterdam : John Benjamins.

VAXELAIRE, Jean-Louis. (1998) Internet : une réelle amélioration du traitement du lexique non-standard? Villetaneuse: Université Paris 13. Mémoire de DEA non-publié.

VAXELAIRE, Jean-Louis. (2005) Les noms propres : Une analyse lexicologique et historique. Paris : Honoré Champion.

VAXELAIRE, Jean-Louis. (2006) "Pistes pour une nouvelle approche de la traduction automatique des noms propres. "Meta 51(4), pp. 719-738

VAXELAIRE, Jean-Louis. (2011) “De Mons à Bergen : De l'intraduisibilité des noms propres. "Translationes 3, pp. 13-28.

VAXELAIRE, Jean-Louis. (à paraître) “Quelles perspectives pour les noms propres ? " À: Lamprou, E. \& J. L. Vaxelaire (éd.). Perspectives en lexicographie. 


\section{BIONOTE / NOTICE BIOGRAPHIQUE}

Jean-Louis Vaxelaire teaches linguistics at the University of Namur, Belgium. His research focuses mainly on proper names (lexicology, lexicography, translation). He is the author of Les Noms propres: Une analyse lexicologique et historique (Champion, 2005), Le désignateur rigide (AFK, to be published soon) and several articles that should be published as a collection next year.

Jean-Louis Vaxelaire enseigne la linguistique à l'Université de Namur en Belgique. Ses travaux de recherche traitent souvent du nom propre (lexicologie, lexicographie, traduction). Il est l'auteur de Les Noms propres : Une analyse lexicologique et historique (Champion, 2005), Le désignateur rigide (AFK, à paraître prochainement) et de divers articles qui devraient être publiés l'année prochaine sous la forme d'un livre. 